\title{
An Enhanced Security Scheme for Wireless Sensor Network Using Mobile Sink Server
}

\author{
Kanagavalli.N,M.E1, Hema Rani.V2 \\ Associate Professor/IT 1, M.E (Software Engineering)2 \\ Saveetha Engineering College, Chennai, India
}

\begin{abstract}
Wireless sensor networks consisting of a large number of low power, low cost sensor node that communicate wirelessly. Such sensor networks can be used in wide range of applications such as military sensing and tracking, health monitoring etc, when the sensing field is too far from the station, transmitting data over long distance using multi-hop may weaken the security strength. To overcome this problem, mobile sinks (MS) are used. Mobile sinks plays a vital role in many wireless sensor applications for efficient data collection and localized sensor reprogramming. MS prolong the lifetime of the sensor network. Security became a critical issue when sensor network with MS are deployed in hostile environment. For providing more security against clone attack and Sybil attack, the proposed scheme implements mobile sink server to offer better security. In Sybil attack, the malicious device illegitimately taking on multiple identities whereas in, clone attack, adversaries may easily capture and compromise sensors and deploy unlimited number of clones of the compromised nodes. Based on the parameters such as bandwidth utilization, speed and time, the malicious act by the adversary are detected. And MS is assigned randomly once it is exposed to threat.
\end{abstract}

Keywords: Distributed. Security, Wireless Sensor networks, AODV, Mobile Sink.

\section{Introduction:}

Wireless sensor networks are potentially one of the most important technologies of this century. Recent advancement in wireless communications and electronics has enabled the development of low-cost, low-power, multifunctional miniature devices for use in remote sensing applications. The combination of these factors has improved the viability of utilizing a sensor network consisting of a large number of intelligent sensors, enabling the collection, processing analysis and dissemination of valuable information gathered in a variety of environments. A sensor network is composed of a large number of sensor nodes which consist of sensing, data processing and communication capabilities. Instead of sending the raw data to the nodes responsible for the fusion, they use their processing abilities to locally carry out simple computations and transmit only the required and partially processed data. Some of the popular applications of sensor network are area monitoring, environment monitoring (such as pollution monitoring), industrial and machine health monitoring, waste water monitoring and military surveillance.

In Mobile Sink Wireless Sensor Networks (MSWSN), all sensors are static other than the sink node. Mobile nodes are the destination of messages originated by sensors, i.e., they represent the endpoints of data collection in the network. They can either autonomously consume collected data for their own purposes or make them available to remote users by using a long range wireless Internet connection. In sensor nodes are static and densely deployed in the sensing area. One or multiple Mobile sinks (MS) move throughout the network to collect data from all sensors. Communication between the source sensors and the MS is either single hop or multi-hop.

During the data collection technique in mobile sink sensor networks, security is an important factor. Node need to be authenticate before start the data collection process. At the same time sensors also need to authenticate the sink. After authentication takes place the start the data communication process with specified rule. During the data collection sensor send their data with encrypting the data packets and send it to the sink node. When sink receive the data it decrypt the packet and check for the adversary modification during data transmission. This node authentication, data encryption and decryption use different cryptography technology. Using cryptography function it secures the communication process.

Mobility is exploited in the field of wireless sensor network to circumvent multi-hop relaying and to reduce energy consumption at nodes near the base station, and hence elongate the lifetime of the network. Mobile elements already exist in the deployment environment; a network node can be attached to these mobile elements for data collection.

Mobile Sink Wireless Sensor Network:

In Mobile Sink Wireless Sensor Networks all the sensors are statically deployed to sense the environment and mobile sink traverse the networks. It overcomes the problem of the sink neighborhood problem. In the sink 
neighborhood problem is neighbor nodes of sink participate more in the data transmission. The result is the faster energy deplete compared to other nodes in the network. If we look over the energy conservation model sensor deplete some amount of energy during the data receiving and the data transmission. As the sensor those are close to the sink, participate more data transmission i.e. for them and for those sensors away from the sink in the same direction.

A malicious node can participate in the data collection process by showing it as the sink node. Then all the sensed data collected by the malicious node, for that we need to authenticate the node before sending the sensed data. If sensors send its packets without encryption then malicious node can accept the packet then it can modify the content of the packet. So we'll lose the original content of the data. Data is neither to be modified nor be dropped. We need to keep data freshness. Otherwise, mobile elements are part of the network infrastructure itself and can be controlled by the network. There exist a number of sensor networks applications that use mobile sinks in their operations, such as data collections in hazardous environments, localize reprogramming, and military navigation. Due to the their operating nature, they often left unattended, hence prone to different kinds of malicious attacks such as the Sybil attacks, clone attacks and wormhole attacks.

\section{System Description And Problem Statement:}

Wireless sensor networks (WSNs) consisting of a large number of low-power, low-cost sensor nodes that communicate wirelessly. Such sensor networks can be used in a wide range of applications, such as, military sensing and tracking, health monitoring, data acquisition in hazardous environments, and habitat monitoring. The sensed data often need to be sent back to the base station for analysis. However, when the sensing field is too far from the base station, transmitting the data over long distances using multi-hop may weaken the security strength (e.g., some intermediate may modify the data passing by, capturing sensor nodes, launching a clone attack, a sybil attack, selective forwarding, sinkhole, and increasing the energy consumption at nodes near the base station, reducing the lifetime of the network. Therefore, mobile sinks (MSs) (or mobile soldiers, mobile sensor nodes) are essential components in the operation of many sensor network applications, including data collection in hazardous environments, localized reprogramming, oceanographic data collection, and military navigation. For the basic probabilistic [17] and q-composite [18] key predistribution schemes, an attacker can easily obtain a large number of keys by capturing a small fraction of the network sensor nodes, making it possible for the attacker to take control of the entire network by deploying a replicated mobile sink, preloaded with some compromised keys to authenticate and then initiate data communication with any sensor node

\subsection{Existing Work:}

In the existing system, the security approach makes the network more resilient to mobile sink replication attacks compared to the single polynomial pool-based key predistribution scheme, it is still vulnerable to stationary access node replication attacks. In these types of attacks, the attacker is able to launch a replication attack similar to the mobile sink replication attack. After a fraction of sensor nodes have been compromised by an adversary, captured static polynomials can be loaded into a replicated stationary access node that transmits the recorded mobile sink's data request messages to trigger sensor nodes to send their aggregated data. It use two separate polynomial pools: the mobile polynomial pool and the static polynomial pool. Polynomials from the mobile polynomial [19] pool are used to establish the authentication between mobile sinks and stationary access nodes, which will enable these mobile sinks to access the sensor network for data gathering. Hence it overcomes mobile sink replication attack [16] and stationary access node replication attack. But it doesn't overcomes clone attack and Sybil attack.

\subsection{Proposed System:}

In the proposed system, Mobile sink servers are implemented to mitigate and over Sybil and clone attack. MS collects data from the sensor node and it is forwarded to MSS and hence to the base station. MSS monitors MS based on parameters such as time, delay and traffic. It act as a guard node incase of Sybil attack and witness node in clone attack. As a guard node it will monitor the traffic and misbehave MS node and sends alert to sensor node, not to forward the sensed data. Thus the mobile sink is revoked from the network and MS is assigned randomly. Clone attack uses RED(Randomized Efficient and Distributed) protocol, witness node will check for the random number, node ID and location ID which is generated with the user information. This system uses AODV routing protocol. 


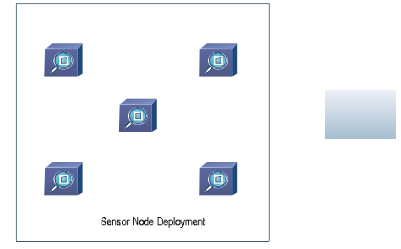

Fig. 1. System Architecture

\subsection{SYBIL ATTACK:}

When a node illegitimately claims multiple identities or claims fake IDs, the WSN suffers from an attack called Sybil attack. The node replicates itself to make many copies to confuse and collapse the network. The system can attack internally or externally. External attacks can be prevented by authentication but not the internal attacks. There should be one to one mapping between identity and entity in WSN. But this attack violates this one-to-one mapping by creating multiple identities.

\subsection{CLONE ATTACK:}

Adversaries may easily capture and compromise sensors and deploy unlimited number of clones of the compromised nodes. Since these clones have legitimate access to the network (legitimate IDs, keys, other security credentials, etc.), they can participate in the network operations in the same way as a legitimate node, and thus launch a large variety of insider attacks or even take over the network. If these clones are left undetected, the network is unshielded to attackers and thus extremely vulnerable. Most existing research efforts in sensor networks against clone attacks focus on preventive technologies rather than detective techniques, e.g., key schemes to prevent sensors from being compromised. Unfortunately, most of these preventive technologies (i.e., key schemes) may easily lose their power against clone attacks [11]. Therefore it is imperative to provide effective/efficient clone attack detection.

\subsection{AODV:}

AODV [8] is an on-demand, single path, loop-free distance vector protocol. It combines the on-demand route discovery mechanism in DSR with the concept of destination sequence numbers from DSDV. However, unlike DSR which uses source routing, AODV takes a hop-by-hop routing approach.

\subsubsection{ROUTE DISCOVERY AND ROUTE MAINTENANCE: \\ 2.5.1.1. ROUTE DISCOVERY:}

In on-demand protocols, route discovery procedure is used by nodes to obtain routes on an 'as needed' basis. In AODV, route discovery works as follows. Whenever a traffic source needs a route to a destination, it initiates a route discovery by flooding a route request (RREQ) for the destination in the network and then waits for a route reply (RREP). When an intermediate node receives the first copy of a RREQ packet, it sets up a reverse path to the source using the previous hop of the RREQ as the next hop on the reverse path. In addition, if there is a valid route available for the destination, it unicasts a RREP back to the source via the reverse path; otherwise, it re-broadcasts the RREQ packet. Duplicate copies of the RREQ are immediately discarded upon reception at every node. The destination on receiving the first copy of a RREQ packet forms a reverse path in the same way as the intermediate nodes; it also unicasts a RREP back to the source along the reverse path. As the RREP proceeds towards the source, it establishes a forward path to the destination at each hop.

\subsubsection{ROUTE MAINTENANCE:}

Route maintenance is done by means of route error (RERR) packets. When an intermediate node detects link failure (via a link-layer feedback, e.g.), it generates a RERR packet. The RERR propagates towards all traffic sources having a route via the failed link, and erases all broken routes on the way. A source upon receiving the RERR initiates a new route discovery if it still needs the route. Apart from this route maintenance mechanism, AODV also has a timer-based mechanism to purge stale routes. 


\section{Proposed Work}

3.1. NODE CREATION AND DATA SENSING:

It contains sensor node creation, mobile sink creation and MSS creation. Sensors within the MCA (Multi-hop Communication Area), called members, must first relay data to the MS which complete the final data transmission to the MSS. Register all the details about all the sensor node under the MCA. Select the sensor nodes from the Multi-hop Communication area and the MS nodes from the Direct Communication Area (DCA). Nodes under single hop comes under DCA. MS collect the data from members and forward it to the MSS.

\subsection{LOCAL MONITORING AND SYBIL ATTACK IDENTIFICATION:}

Local monitoring is a collaborative detection strategy where a node monitors the traffic going in and out of its neighbors. For a node, say a to be able to watch a node, say N2 a must be a neighbor of both N2 and the previous hop fromN2, say N1.We call a is a guard node for N2 over the link N1 ->N1. Information from each packet sent from $\mathrm{X}$ to $\mathrm{A}$ is saved in a watch buffer at each guard. The guards expect that $\mathrm{A}$ will forward the packet toward the ultimate destination, unless $\mathrm{A}$ is itself the destination. Each entry in the watch buffer is time stamped with a time threshold, by which A must forward the packet. Each packet forwarded by A with X as a previous hop is checked for the corresponding information in the watch buffer. The check can be to verify if the packet is fabricated or duplicated (no corresponding entry in the buffer), corrupted (no matching hash of the payload), dropped, or delayed (entry is not matched within T).

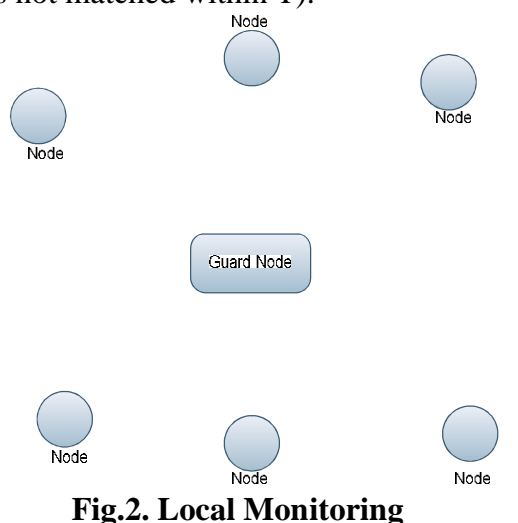

\subsection{MITIGATING SYBIL ATTACK:}

The basic idea is to extend the knowledge at each guard to include the identity of the next hop for the packet being relayed. This additional knowledge can be collected during route establishment. The routing protocols require modification to the protocol to build the next-hop information at the guards. Examples of these protocols are the reactive routing protocols that use control packet flooding of route requests (REQs) and route replies (REPs) to establish the route between the source and the destination. In these protocols, when a source node desires to send a message to some destination node and does not already have a valid route to that destination, it initiates a route discovery process to locate the other node. It broadcasts a route request packet to its neighbors, which then forward the request to their neighbors, and so on, until either the destination or an intermediate node with a "fresh enough" route to the destination is located. Along with its own sequence number and the broadcast ID, the source node includes in the REQ the most recent sequence number it has for the destination. During the process of forwarding the REQ, intermediate nodes record in their route tables the address of the neighbor from which the first copy of the broadcast packet is received, thereby establishing a reverse path. Once the REQ reaches the destination, the destination node responds by unicasting a route reply packet back to the neighbor from which it first received the REQ. As the REP traverses along the reverse path, nodes along this path set up forward route entries in their route tables which point to the node from which the REP came. The changes to the basic version of AODV (Ad hoc On-Demand Distance Vector) to enable the guards to build the necessary knowledge for detecting the misrouting attack. The idea behind the solution is to augment the additional information required for detection to the control traffic responsible for route establishment and require the guards to collect that information during the route establishment phase. To collect the next-hop identity information in AODV, the forwarder of the REQ attaches the previous two hops to the REQ packet header. 


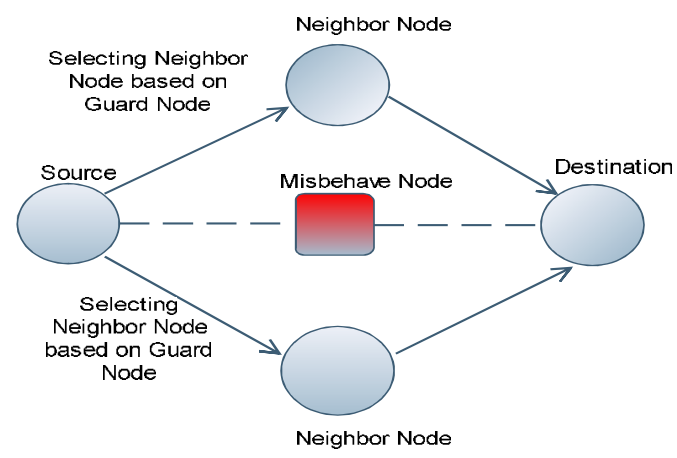

Fig.3. Mitigating misbehave node

\subsection{IDENTIFYING AND RESOLVING CLONE ATTACK:}

Using RED protocol, Witness node will check the Random number, Node ID, Location ID which is generated with the user information. If the witness node receives two different incoherent locations for a same identity node, then it results in clone detection. The cloned node will be revoked from the network by the mobile sink server. RED executes at fixed intervals of time. Every run of the protocol consists of two steps. In the first step a random value, rand, is shared among all the nodes . This can be performed with centralized broadcasting (for example, from a satellite or other kinds of ground-based central stations), or with distributed mechanisms . In the second step (i.e., the detection phase), each node digitally signs and broadcasts its claim: ID and geographic location. For each node, each of its $d$ neighbors sends (with probability $p$ ) the claim to a set of $g \geq 1$ pseudo-randomly selected network locations. It avoid sending the claim to a specific node ID because this kind of solution needs more information to scale. More than one node can witness a clone attack. However, note that RED could easily be modified to generate more than one witness.

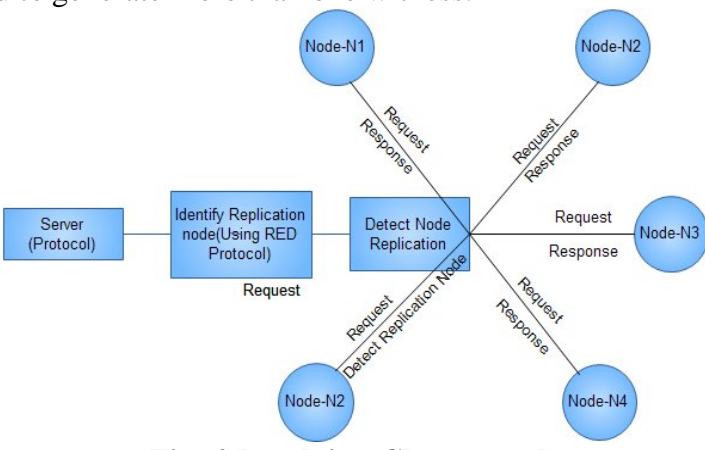

Fig. 4.Resolving Clone attack

\subsection{Throughput/ Delivery Ratio}

\section{Performance Metrics:}

In Wireless Sensor Networks throughput is the average rate of successful message delivery over communication radio. This data may be delivered by the physical or logical link, or pass through certain network nodes. The throughput is usually calculated in bits per second (bps), and sometimes in data packets per second or data packets per time slot. Yuxi et al. [12] showed that lossy links do have significant impact on the maximum achievable throughput. There are some cases, where a network can achieve half of the throughput of the corresponding lossless network. Lossy links also affects energy efficiency. Lossy network can only achieve half of the throughput when links are lossless.

\subsection{Network Life Time}

Network lifetime is the key characteristic for evaluating sensor networks in an application specific way. The lifetime of sensor network depends on the operation time of individual sensor nodes. Lifetime of wireless sensor networks ends when first node dies in the network. Y. Chen et al. [7] described two key parameters at the physical layer that affect the lifetime of the network: the state of the channel and the residual energy of sensors. Here in this letter they proposed a greedy approach to lifetime maximization which achieves considerable improvement in the lifetime performance. 


\subsection{Data Freshness}

In [14] Given that all sensor networks stream some forms of time varying measurements, it is not enough to guarantee confidentiality and authentication; we also must ensure each message is fresh. Informally, data freshness denotes that the data is recent, and it confirms that no adversary replayed old messages. We identify two types of freshness: weak freshness, which provides partial message ordering, but carries no delay information, and strong freshness, which provides a total order on a request response pair, and allows for delay estimation [14]. Weak freshness is required by sensor measurements, while strong freshness is useful for time synchronization within the network [15].

\section{Conclusion:}

In this paper, an enhanced security scheme for wireless sensor network using mobile sink is implemented against Sybil attack and clone attack. The proposed scheme is based on mobile sink server which determines the parameters such as traffic, time and bandwidth of all the mobile sink. An uncompromised mobile node should never move at speeds in excess of the system-configured maximum speed. If the node misbehaves it revokes and assign MS randomly. Thus the replication of node and its identity can be resolved. Hence data collection can be done in secure manner. All the simulation has been carried out with NS 2.34. This thesis is supported by the literature survey in the area of Mobile Sink Wireless Sensor Networks to make it complete.

\section{References}

[1] I.F. Akyildiz, W. Su, Y. Sankarasubramaniam, and E. Cayirci, "Wireless sensor networks: A survey," The International Journal of Computer and Telecommunications Networking Computer Networks, vol. 38, no. 4, pp. 393-422, March 2002.

[2] I. Chatzigiannakis, A. Kinalis, and S. Nikoletseas, "Sink mobility protocol for data collection in wireless sensor networks," Proc. of the 4th ACM International Workshop on Mobility Management and Wireless Access (MOBIWAC'06), pp. 52-59, 2006.

[3] S. Basagni, A. Carosi, E. Melachrinoudis, C. Petrioli, and Z. M. Wang, "Protocols and model for sink mobility in wireless sensor networks," ACM SIGMOBILE Mobile Computing and Communications Review, vol. 10, no. 4, pp. 28-30, 2006.

[4] L. Cheng, Y. Chen, C. Chen, and J. Ma, "Query-based data collection in wireless sensor networks with mobile sinks," Proc. of the 2009 International Conference on Wireless Communications and Mobile Computing: Connecting the World Wirelessly, pp. 1157$1162,2009$.

[5] B. Parno, A. Perrig, and V. D. Gligor, "Distributed detection of node replication attacks in sensor networks," Proc. of the 2005 IEEE Symposium on Security and Privacy (S\&P'05), pp. 49-63, 2005.

[6] W. Du, J. Deng, Y. S. Han, and P. K. Varshney, "A pairwise key pre-distribution scheme for wireless sensor networks," In ACM CCS 2003, pages 42\{51,Oct. 2003\}.

[7] Yunxia Chen and Qing Zhao "On the Lifetime of Wireless Sensor Networks," IEEE communications letters, vol. 9, no. 11, pp. 976978, November 2005.

[8] Charles E. Perkins and Elizabeth M. Royer, "Ad-hoc On-Demand Distance Vector Routing," Proceeding of the 2nd IEEE Workshop on Mobile Computing Systems and Applications (WMCSA'99), New Orleans, LA, USA, February 1999, pages 90-100.

[9] Murat Demirbas and Youngwhan song, "An RSSI-based Scheme for Sybil Attack Detectionin Wireless Sensor Networks,"Proceeding of the 2006 International symposium on World of Wireless, Mobile and Multimedia Networks (WOWMOM'06), pages 564-570.

[10] Kai Xing, Fang Liu, Xiuzhen Cheng, David H.C. Du, "Real-Time Detection of Clone Attacks in Wireless Sensor Networks," icdcs, pp.3-10, 2008 The 28th International Conference on Distributed Computing Systems, 2008.

[11] H. Choi, S. Zhu, and T. Laporta. Set: Detecting node clones in sensor networks. In SecureComm'07, 2007.

[12] Li, Harnes, Holte, "Impact of Lossy Links on Performance of Multihop Wireless Networks," IEEE, Proceedings of the 14th International Conference on Computer Communications and Networks, pp. 303 - 308, Oct 2005.

[13] Amar Rasheed and Rabi N. Mahapatra, "The Three-Tier Security Scheme in Wireless Sensor Networks with Mobile Sinks," Proceedings of the IEEE Transactions on parallel and distributed systems, VOL. 23, NO. 5, MAY 2012.

[14] Adrian Perrig, Robert Szewczyk, Victor Wen, David Culler and J. D. Tygar, "SPINS: Security Protocols for Sensor Networks," Wireless Networks, pp. 189-199, 2001.

[15] Apostolos, Pyrgelis. "Cryptography and Security in Wireless Sensor Networks," Department of Computer Engineering and Informatics, 2009.

[16] J. R. Douceur, "The Sybil attack," In First International Workshop on Peer-to-Peer Systems (IPTPS '02), Mar. 2002.

[17] L. Eschenauer and V.D. Gligor, "A Key Management Scheme for Distributed Sensor Networks," Proc. ACM Conf. Computer Comm. Security (CCS '02), pp. 41-47, 2002.

[18] H. Chan, A. Perrig, and D. Song, "Random Key Pre-Distribution Schemes for Sensor Networks," Proc. IEEE Symp. Research in Security and Privacy, 2003.

[19] Y. Tirta, Z. Li, Y. Lu, and S. Bagchi, "Efficient Collection of Sensor Data in Remote Fields Using Mobile Collectors," Proc. 13th Int'l Conf. Computer Comm. and Networks (ICCCN'04), Oct.2004. 